\title{
Ability of the Premise Condition Index to Identify Premises with Adult and Immature Aedes Mosquitoes in Kampong Cham, Cambodia
}

\author{
John Hustedt, ${ }^{1,2 \star}$ Dyna Doum, ${ }^{1}$ Vanney Keo, ${ }^{1}$ Sokha Ly, ${ }^{3}$ BunLeng Sam, ${ }^{3}$ Vibol Chan, ${ }^{4}$ Sebastien Boyer, ${ }^{5}$ Marco Liverani, ${ }^{2}$ \\ Neal Alexander, ${ }^{2}$ John Bradley, ${ }^{2}$ Didot Budi Prasetyo, ${ }^{6}$ Agus Rachmat, ${ }^{6}$ Sergio Lopes, ${ }^{1}$ Rithea Leang, ${ }^{3}$ and Jeffrey Hii ${ }^{1}$ \\ ${ }^{1}$ Malaria Consortium, Phnom Penh Center, Phnom Penh, Cambodia; ${ }^{2}$ London School of Hygiene and Tropical Medicine, London, United \\ Kingdom; ${ }^{3}$ Cambodian National Dengue Control Program, Phnom Penh, Cambodia; ${ }^{4}$ World Health Organization, Phnom Penh, Cambodia; \\ ${ }^{5}$ Institut Pasteur du Cambodge, Phnom Penh, Cambodia; ${ }^{6}$ US Naval Medical Research Unit-2, Phnom Penh, Cambodia
}

\begin{abstract}
Aedes-transmitted diseases, especially dengue, are increasing throughout the world and the main preventive methods include vector control and the avoidance of mosquito bites. A simple Premise Condition Index (PCI) categorizing shade, house, and yard conditions was previously developed to help prioritize households or geographical areas where resources are limited. However, evidence about the accuracy of the PCl is mixed. The current study aimed to contribute to a better understanding of the relevance by collecting data from 2,400 premises at four time points over 1 year in Kampong Cham, Cambodia. Regression models were then used to identify associations between PCl and $A e d e s$ adult female mosquitoes and pupae. In addition, receiver operating characteristic curves were used to measure the ability of $\mathrm{PCl}$ to identify premises in the top quartile of mosquito abundance. The density of adult Aedes females was positively associated with $\mathrm{PCl}$ at the household (ratio of means $=1.16$ per point on the $\mathrm{PCl}$ scale) and cluster level (ratio of means = 1.54). However, the number of Aedes pupae was negatively associated with $\mathrm{PCl}$ at the household level (rate ratio $=0.74$ ) and did not have a statistically significant association at the cluster level. Receiver operating characteristic curves suggest the PCl score had "rather low accuracy" (area under the ROC curve $=0.52$ and 0.54 ) at identifying top-quartile premises in terms of adult female Aedes and pupae, respectively. These results suggest that caution is warranted in the programmatic use of $\mathrm{PCl}$ in areas of similar geography and mosquito abundance.
\end{abstract}

\section{INTRODUCTION}

Dengue is the most rapidly spreading mosquito-borne viral disease in the world and is caused by bites of infected Aedes mosquitoes, principally Aedes aegypti. ${ }^{1}$ Dengue is endemic worldwide, with a high concentration in the Asian region, which shoulders $70 \%$ of the global disease burden. Although a number of promising vaccine candidates are in preclinical and clinical development, ${ }^{2}$ methods of genetic control of mosquitoes are being developed, ${ }^{3,4}$ and Wolbachia-infected mosquitoes show promise, ${ }^{5,6}$ these interventions are unlikely to eliminate dengue on their own. ${ }^{7}$ Therefore, vector control will remain a key component of dengue control in the short and medium term.

One important aspect of vector control is the elimination of the most productive breeding sites. ${ }^{8}$ For example, one study in Australia found that one well and one rainwater tank were responsible for $28 \%$ of all immature larvae out of 1,349 premises inspected. ${ }^{9}$ Similarly, in Cambodia, large water jars, drums, and concrete tanks were found to harbor $90 \%$ of the pupal biomass. ${ }^{10}$ In addition, studies documented that particularly high levels of Aedes productivity can be found in "key premises," ${ }^{11-14}$ defined as those with three or more positive containers. ${ }^{9}$ In Australia, $1.9 \%$ of premises accounted for $47.2 \%$ of positive containers. ${ }^{9}$ In Ecuador, $11 \%$ of households contained $81.7 \%$ of pupae during the rainy season and $5 \%$ of households contained $80 \%$ of pupae during the dry season. ${ }^{12}$ Thus, it is clear that the identification of key premises is crucial to inform vector control operations - an activity which can be conducted through pupal/demographic surveys of household water containers.

*Address correspondence to John Hustedt, Malaria Consortium, Phnom Penh Center, St. Sothearos, Bldg. "H," 1st Floor, Rm. No. 192, Phnom Penh 12301, Cambodia. E-mail: john.hustedt@|shtm.ac.uk
However, the ubiquity of water containers tends to make pupal/demographic surveys laborious. ${ }^{15}$ Therefore, additional methods have been explored to identify key premises without needing to do extensive pupal/demographic surveys, or enter premises, because owners refusing access to premises has been reported as a key challenge. ${ }^{16}$ The Premise Condition Index $(\mathrm{PCl})$ is one such approach that could help prioritize outbreak response in terms of Aedes infestation risk. ${ }^{9}$ This index evaluates the shade, house, and yard conditions of premises to produce risk strata. In addition to targeting treatment of key premises, this method could potentially be used to prioritize villages or other geographical areas when funding or human resources are insufficient to treat all outbreak areas.

Existing evidence of the value of the $\mathrm{PCl}$ to inform vector control programs is mixed. The $\mathrm{PCl}$ was first described and evaluated in Queensland, Australia, where it was found that inspecting $9.5 \%$ of premises with a high $\mathrm{PCl}$ score of 8-9 (out of 9) identified $54.4 \%$ of infested premises. Comparison of highest to lowest scores indicated a risk of infestation 5.6 times higher, with the number of positive containers 14.3 times higher. ${ }^{9}$ Other studies found a correlation between $\mathrm{PCl}$ and the number of positive containers ${ }^{17-21}$ and/or positive premises. ${ }^{19,20,22}$ Premise Condition Index has also been used to create risk strata, where a positive correlation $(r=0.968, P<0.01)$ was identified in Brazil between risk strata and houses positive for Aedes albopictus eggs. ${ }^{23}$ By contrast, other studies found no association of Aedes mosquitoes with PCl. ${ }^{24,25}$ Furthermore, serious limitations or missing information exist in many of the past studies. Some studies report associations but do not provide data related to $\mathrm{PCI}$ in their article $18,21,24,26,27$ or relied on low sample sizes with wide Cls. ${ }^{19}$

Considering these uncertainties, this study aimed to assess whether higher mean densities of adult female Aedes mosquitoes and Aedes pupae are associated with worse premise conditions, as measured by PCl; and whether this association 
leads to reliable predictions of which premises should be targeted for interventions. The study was conducted in Cambodia, a country with one of the highest per capita incidence rates in Asia, at 0.7-3.0 per 1,000 population per year, ${ }^{28,29}$ and recurring outbreaks every $3-5$ years. ${ }^{30}$ The Cambodia National Dengue Control Program developed a protocol to respond to outbreaks, defined as three or more cases in one village per year, which includes applying larvicides (e.g., temephos), adulticides (e.g., thermal fogging with pyrethroids), and distributing information, education, and communication materials. These activities are implemented throughout the entire outbreak villages and can require significant financial and human resources, especially if distances between villages and the number of outbreaks are large. In this setting, if shown to be effective, $\mathrm{PCl}$ could potentially be used to prioritize interventions when funds are insufficient to treat all houses or geographic areas. An advantage of the index is that it can be completed quickly and there is no need to enter houses. Although previously published evidence on the relevance of the $\mathrm{PCl}$ varies by geography and mosquito life stage, no studies and field evaluations have previously been reported from Cambodia or Southeast Asia.

\section{MATERIALS AND METHODS}

Study setting. The data used in this study were collected during a cluster randomized trial on the effect of guppy fish and pyriproxyfen on entomological outcomes, ${ }^{31}$ conducted in 30 clusters in two operational districts within Kampong Cham province. Each cluster had an average of approximately 200 households or 1,000 individuals and included one or more villages that were separated by neighboring villages by at least $200 \mathrm{~m}$. Kampong Cham has one of the highest dengue incidence rates in Cambodia (1.6 cases per 1,000 people per year) and the environmental characteristics are similar to most dengue-endemic areas of Cambodia $(\mathrm{H}$. Ra, personal communication). The dry season lasts from December to April, the light rain season from April to July, and the heavy rain season from August to October. This study only uses data from the pre-intervention baseline surveys and control clusters, which did not receive an intervention, of the aforementioned trial and are considered to be more representative of the typical conditions in the area. More detailed information about the study site can be found in the trial protocol. ${ }^{31}$

Outcomes. The primary outcome was the association between PCl (defined below) and the mean density of adult female $A$. aegypti at household level. Secondary outcomes include the association between 1) $\mathrm{PCl}$ and the mean density of adult female $A$. aegypti at cluster level and 2) association between $\mathrm{PCl}$ and the number of Aedes pupae per household and per cluster.

Mosquito collection and PCI scoring. Data were collected at four time points covering all three main seasons: survey 1 was in October/November 2015 during the rainy season, survey 2 was in February/March 2016 during the dry season, survey 3 was in May/June 2016 during the light rain season, and survey 4 was in September/October 2016 in the heavy rain season. The survey methodology was developed following the WHO guidelines for entomological collections. ${ }^{30}$ The survey team consisted of experienced government staff who received 3 days training before the start of the surveys. Twenty surveyors completed each of the four entomology surveys within 20 days. All tools and materials were pretested during training. Houses within each cluster were selected using a random-number generator applied to the village list managed by the village head.

Larvae and pupae collection were completed using the fivesweep net method ${ }^{15}$ for containers larger than $50 \mathrm{~L}$. For this method, a net of size $20 \mathrm{~cm}$ by $33 \mathrm{~cm}$ was used. Surveyors turned the net in an anti-clockwise manner five times, then waited 1 minute and performed one sweep from the bottom. This method can sample around $35 \%$ of larvae and $31 \%$ of pupae, and the total number estimated by an adjustment factor. ${ }^{15}$ For containers of less than $50 \mathrm{~L}$, all the water was poured through the sweep net. All containers within selected households were inspected. All pupae and larvae were put in a plastic bag, labeled, and taken back to the provincial laboratory for identification to the species level for Aedes, otherwise to genus.

The adult resting catch was completed using a batterypowered, portable aspirator (Camtech, Phnom Penh, Cambodia) for 10 minutes per house in the bedrooms and living spaces, starting in the bedroom and aspirating up and down the wall (from floor to $1.5 \mathrm{~m}$ ) around the home in a clockwise manner. The mosquitoes were kept in a screw-top container inside a cold box and transported to the provincial laboratory for identification to the species level for Aedes, otherwise to genus. All adult Aedes mosquitoes were sexed. After identification, all mosquitoes were taken to the United States Naval Medical Research Unit-2 in Phnom Penh where entomologists confirmed identification of a random sample of $50 \%$ of immature and adult mosquitoes. Each house in the survey was scored on the degree of shade, condition of house, and condition of yard according to the method developed by TunLin et al. ${ }^{9}$ Each category is scored from 1 to 3 , and the sum represents the $\mathrm{PCl}$ score. The teams were provided with objective measures for scoring in each category (see Table 1), a laminated sheet including pictures of example premises for each score, and given training to standardize scoring between the three teams. In addition, a fourth category representing the source of water was scored; however, because of the homogeneity of water infrastructure, the results are not reported here.

Climate. General climate data (rainfall, temperature, and humidity) were recorded at one of the intervention health centers using a rain gauge and a $\mathrm{Hobo}^{\mathrm{TM}}$ onset data logger (Onset Computer Corporation, Bourne, MA) (all villages included in the study have virtually the same climate). Data from the all United States National Aeronautics and Space Administration satellites on climate are also available to double check the accuracy of these measurements.

Sample size. Sample size was determined for the needs of the corresponding trial and is discussed in length in the protocol. ${ }^{31}$ However, the sample size is at least as large as four other studies which reported a significant association or correlation of $\mathrm{PCl}$ with houses or containers with Aedes mosquitoes. ${ }^{19-21,32}$

Statistical analysis. All analyses were performed in $\mathrm{R}$ Studio version 3.5.0 (Murray Hill, NJ) and Stata ${ }^{\circledR}$ version 14.2 (College Station, TX). The association between Aedes density and $\mathrm{PCl}$ was assessed through negative binomial regression using the number of adults per household as the response and a logarithmic link function. Hence, this analysis yields density ratios as an outcome measure. Models combined data from all seasons and included survey as a fixed effect term. Additional models including an interaction term of survey and $\mathrm{PCl}$ were 
TABLE 1

Measures for scoring the Premise Condition Index

\begin{tabular}{|c|c|c|}
\hline Premise variables & Description & Classification score \\
\hline \multirow[t]{3}{*}{ P1. House condition } & a. Well-maintained, e.g., newly painted or new house & 1 \\
\hline & b. Moderately well-maintained house & 2 \\
\hline & $\begin{array}{l}\text { c. Not well-maintained house, e.g., paint peeling, broken items visible, } \\
\text { dilapidated old house }\end{array}$ & 3 \\
\hline \multirow[t]{3}{*}{ P2. Yard condition } & $\begin{array}{l}\text { a. Tidy yard, e.g., no rubbish or trash evident, well-maintained } \\
\text { gardens, and lawn }\end{array}$ & 1 \\
\hline & b. Moderately tidy yard & 2 \\
\hline & $\begin{array}{l}\text { c. Untidy yard, rubbish and trash abundant, and the garden or lawn } \\
\text { with overgrown grass }\end{array}$ & 3 \\
\hline \multirow[t]{3}{*}{ P3. Shade condition } & a. Very little or no shade $(<25 \%)$, e.g., no major trees or bush & 1 \\
\hline & b. Some shade $(>25 \%$ but $<50 \%)$ & 2 \\
\hline & $\begin{array}{l}\text { c. Plenty of shade, }>50 \% \text {, e.g., large trees evident, layers of shrubs, } \\
\text { green house, plastic tarp sheet, or overhanging roofs used }\end{array}$ & 3 \\
\hline \multirow[t]{3}{*}{ P4. Water supply and storage } & a. Piped water supply only & 1 \\
\hline & b. Well water supply only & 2 \\
\hline & c. Rainwater and/or river water & 3 \\
\hline
\end{tabular}

also run. A likelihood ratio test showed the interaction term to not be statistically significant $(P=0.07)$, and therefore, the model with interaction was not included in the results. A similar model was used for the secondary outcomes, with the numbers of pupae, rather than adults, as the response. Additional zero-inflated models were fitted; however, the model fit better without zero inflation. All models used the robust sandwich estimator of standard errors ${ }^{33}$ to account for correlation of responses within clusters.

Associations between $\mathrm{PCl}$ and vector density are necessary but not sufficient for $\mathrm{PCl}$ to have sufficient sensitivity and specificity to be efficient in practice. Receiver operating characteristic (ROC) curves were used to ascertain the ability of $\mathrm{PCl}$ to predict the premises in the top quartile of mosquito biomass. Their accuracy was classified according to the value of the area under the ROC curve (AUC): not informative (AUC $\leq 0.5$ ), rather low accuracy $(0.5<A \cup C \leq 0.7)$, accuracies useful for some purposes $(0.7<A \cup C \leq 0.9)$, and rather high accuracy $\left(0.9<\right.$ AUC) ${ }^{34}$

Ethical approval. Ethical clearance was received by the Cambodian National Ethics Committee for Health Research on October 9, 2014 (ethics reference number 0285). In addition, ethics approval was received from the London School of Hygiene and Tropical Medicine Observational/Interventions Research Ethics Committee (ethics reference number 8812).

\section{RESULTS}

During the study period, a total of 2,400 premises were inspected for the presence of immature and adult Aedes and assigned $\mathrm{PCl}$ scores. The average monthly rainfall during the study was $11 \mathrm{~mm}$ during the dry season (December-April), $139 \mathrm{~mm}$ during the light rain season (May-July), and 276 during the heavy rain seasons (August-November). As reported in Table 1, the majority of premises (89\%) were assigned a PCl score between 5 and 7 , and only $3 \%$ and $0.4 \%$ were assigned a $\mathrm{PCl}$ score of 8 or 9 , respectively. The median of each component variable of the $\mathrm{PCl}$ was 2 , and all possible values (1, 2 and 3 ) were observed for each component. This suggests that the overall $\mathrm{PCl}$ was not being dominated by any single component.

Distribution of adult female Aedes mosquitoes by PCI ranking. Table 2 shows $26 \%$ of houses overall had some adult female Aedes, with an average of 0.56 each (SD 2.18, range 0-82). The percentage of positive houses and Aedes females per house increased during the light rain season to $58 \%$ and 1.88 (SD 4.81, range 0-82), respectively. The percentage of houses positive for Aedes females varied among overall PCl scores (17-33\%) and among different seasons (17-58\%). The average number of Aedes females per house also varied widely among overall PCl scores (0.21-0.73) and over seasons (0.24-1.88). The highest numbers of positive houses and average number of adult female Aedes was among premises with $\mathrm{PCl}$ scores of 6 and 7 . Table 3 shows that $46 \%$ of premises and $15 \%$ of containers were positive for Aedes pupae and/or larvae with an average of seven pupae per house. The proportion of positive premises varied quite widely between $\mathrm{PCI}$ scores (22-51\%) and between surveys (36-71\%) with light rain (peak) season having by far the highest proportion of positive houses (71\%). The percent of containers positive for larvae or pupae also varied among $\mathrm{PCl}$ scores (7-20\%) and surveys (10-21\%). Only $1 \%$ of premises received a $\mathrm{PCl}$ score of three and a few of those premises had extremely high numbers of Aedes pupae. The particular reason for the large number of pupae is that two premises had a large water container used for animal husbandry that were not often cleaned and held hundreds of pupae. Table 4 shows the results of the negative binomial regression models for adult female Aedes mosquitoes. The model including two dependent variables (PCI scores and survey) was found to fit best. The number of adult Aedes females was positively associated with PCl (rate ratio $[\mathrm{RR}]$ per point $=1.16,95 \% \mathrm{Cl}$ : $1.02-1.31$ ). A cluster-level model of adult Aedes females by cluster had a slightly higher RR, although wider Cls (RR=1.54, 95\% Cl: 1.11-2.08).

Correlation of immature Aedes mosquitoes with PCI. Table 4 also shows the results of negative binomial regression models for Aedes adults and pupae. At the house level, the number of pupae were statistically significantly negatively associated with $\mathrm{PCl}$ scores (RR $=0.74,95 \% \mathrm{Cl}$ : 0.59-0.93), whereas the number of adult Aedes were significantly positively associated with $\mathrm{PCl}$ scores (RR $=1.16,95 \% \mathrm{Cl}$ : $1.02-1.31)$. The model investigating the correlation between number of Aedes pupae and $\mathrm{PCl}$ was not significant at the cluster level, whereas the model for adults was positively associated ( $R R=1.52,95 \% \mathrm{Cl}$ : $1.11-2.08)$.

Receiver operating characteristic curve analysis for predicting the top quartile of adult Aedes mosquitoes. The $\mathrm{PCl}$ score was considered to have "rather low accuracy" predicting premises in the top quartile of adult female Aedes mosquitoes, with an AUC of 0.54 (95\% Cl: 0.52-0.56, Figure 1). A cut point of 5 had high sensitivity (94\%) and low specificity 
TABLE 2

Adult female Aedes indicators by Premise Condition Index (PCl) ranking over different seasons

\begin{tabular}{|c|c|c|c|c|}
\hline $\mathrm{PCl}$ score & Number of houses (\%) & $\begin{array}{l}\text { Houses with at least } \\
\text { one Aedes female (\%) }\end{array}$ & Aedes females & $\begin{array}{l}\text { Mean Aedes females per house } \\
\text { (SD, minimum-maximum) }\end{array}$ \\
\hline \multicolumn{5}{|c|}{ All time points combined ( $n=30$ clusters) } \\
\hline 3 & $30(1)$ & $5(17)$ & 11 & $0.37(1.03,0-5)$ \\
\hline 4 & $138(6)$ & $32(23)$ & 50 & $0.36(0.85,0-6)$ \\
\hline 5 & $623(26)$ & $133(21)$ & 224 & $0.36(0.93,0-8)$ \\
\hline 6 & $1,178(49)$ & 329 (28) & 791 & $0.67(2.78,0-82)$ \\
\hline 7 & $327(14)$ & $97(30)$ & 239 & $0.73(2.16,0-30)$ \\
\hline 8 & $71(3)$ & $15(21)$ & 22 & $0.31(0.77,0-4)$ \\
\hline 9 & $9(0)$ & $3(33)$ & 3 & $0.33(0.50,0-1)$ \\
\hline Missing & $24(1)$ & $4(17)$ & 5 & $0.21(0.51,0-2)$ \\
\hline Total & $2,400(100)$ & $618(26)$ & 1,345 & $0.56(2.18,0-82)$ \\
\hline \multicolumn{5}{|c|}{ October 2015 (heavy rain season): control at baseline ( $n=10$ clusters) } \\
\hline 3 & $3(1)$ & $0(0)$ & 0 & $0.00(0,0-0)$ \\
\hline 4 & $31(8)$ & $8(26)$ & 10 & $0.32(0.60,0-2)$ \\
\hline 5 & $126(32)$ & $23(18)$ & 38 & $0.30(0.79,0-5)$ \\
\hline 6 & $183(46)$ & $25(14)$ & 33 & $0.18(0.52,0-3)$ \\
\hline 7 & $41(10)$ & $11(27)$ & 19 & $0.46(1.07,0-6)$ \\
\hline 8 & $11(3)$ & $1(9)$ & 1 & $0.09(0.30,0-1)$ \\
\hline 9 & $0(0)$ & $0(0)$ & 0 & - \\
\hline Missing & $5(1)$ & $0(0)$ & 0 & $0.00(0,0-0)$ \\
\hline Total & $400(0)$ & $68(17)$ & 101 & $0.25(0.69,0-6)$ \\
\hline \multicolumn{5}{|c|}{ February 2016 (dry season) ( $n=10$ clusters) } \\
\hline 3 & $3(1)$ & $1(33)$ & 5 & $1.67(2.89,0-5)$ \\
\hline 4 & $14(4)$ & $4(29)$ & 7 & $0.50(1.09,0-4)$ \\
\hline 5 & $187(47)$ & $42(22)$ & 71 & $0.38(1.03,0-8)$ \\
\hline 6 & $161(40)$ & $47(29)$ & 106 & $0.66(1.62,0-14)$ \\
\hline 7 & $23(6)$ & $6(26)$ & 7 & $0.30(0.56,0-2)$ \\
\hline 8 & $6(2)$ & $1(17)$ & 1 & $0.17(0.41,0-1)$ \\
\hline 9 & $3(1)$ & $0(0)$ & 0 & $0.00(0,0-0)$ \\
\hline Missing & $3(1)$ & $0(0)$ & 0 & $0.00(0,0-0)$ \\
\hline Total & $400(100)$ & $101(25)$ & 197 & $0.49(1.29,0-14)$ \\
\hline \multicolumn{5}{|c|}{ June 2016 (light rain season) ( $n=10$ clusters) } \\
\hline 3 & $4(1)$ & $1(25)$ & 1 & $0.25(0.50,0-1)$ \\
\hline 4 & $32(8)$ & $10(31)$ & 22 & $0.69(1.35,0-6)$ \\
\hline 5 & $54(14)$ & $29(54)$ & 64 & $1.19(1.63,0-7)$ \\
\hline 6 & $230(58)$ & $148(64)$ & 505 & $2.20(5.8,0-82)$ \\
\hline 7 & $78(20)$ & $42(54)$ & 160 & $2.05(3.93,0-30)$ \\
\hline 8 & $2(1)$ & $0(0)$ & 0 & $0.00(0,0-0)$ \\
\hline 9 & $0(0)$ & $0(0)$ & 0 & - \\
\hline Missing & $0(0)$ & $0(0)$ & 0 & - \\
\hline Total & $400(100)$ & $230(58)$ & 752 & $1.88(4.81,0-82)$ \\
\hline \multicolumn{5}{|c|}{ October 2016 (heavy rain season) ( $n=10$ clusters) } \\
\hline 3 & $4(1)$ & $1(25)$ & 1 & $0.25(0.5,0-1)$ \\
\hline 4 & $13(3)$ & $2(15)$ & 2 & $0.15(0.38,0-1)$ \\
\hline 5 & $42(11)$ & $11(26)$ & 14 & $0.33(0.61,0-2)$ \\
\hline 6 & $280(70)$ & $50(18)$ & 68 & $0.24(0.59,0-3)$ \\
\hline 7 & $56(14)$ & 7 (13) & 9 & $0.16(0.46,0-2)$ \\
\hline 8 & $4(1)$ & $2(50)$ & 2 & $0.50(0.58,0-1)$ \\
\hline 9 & $1(0)$ & $0(0)$ & 0 & $0.00(0,0-0)$ \\
\hline Missing & $0(0)$ & $0(0)$ & 0 & - \\
\hline Total & $400(100)$ & $73(18)$ & 96 & $0.24(0.57,0-3)$ \\
\hline
\end{tabular}

(7\%), whereas 7 had low sensitivity (19\%) and high specificity (83\%). For clusters, the $\mathrm{PCl}$ score was also considered to have "rather low accuracy," with an AUC of 0.64 (95\% Cl: 0.44-0.80, Figure 2). No cut point for either curve gives an adequate combination of sensitivity and specificity.

Receiver operating characteristic curve analysis for predicting the top quartile of Aedes pupae. The PCl score was considered to have "rather low accuracy" when predicting premises in the top quartile for Aedes pupae, with an AUC of 0.52 (95\% Cl: $0.50-0.54$, Figure 3$)$. A cut point of 5 had high sensitivity (93\%) and low specificity $(7 \%)$, whereas 7 had low sensitivity (16\%) and high specificity (83\%). For clusters, the $\mathrm{PCl}$ score was again considered to have "rather low accuracy" when predicting the clusters in the top quartile for Aedes pupae, with an AUC of 0.62 (95\% Cl: 0.44-0.80, Figure 4). No cut point for either curve gives an adequate combination of sensitivity and specificity. This low degree of accuracy is consistent with the negative association presented earlier.

\section{DISCUSSION}

The PCl was found to be weakly associated with the density of adult female Aedes at the household and cluster level, and negatively associated with the number of Aedes pupae at the household level. Therefore, our hypothesis that higher mean densities of Aedes would be associated with worse premise conditions was correct for adult females, but not for pupae. The five premises with the highest number of Aedes pupae represented $25 \%$ of the total pupae and had relatively lower $\mathrm{PCl}$ scores (one house had a $\mathrm{PCl}$ of 3 ). This may have been 
TABLE 3

Immature Aedes indicators by Premise Condition Index $(\mathrm{PCl})$ ranking over different seasons

\begin{tabular}{|c|c|c|c|c|c|c|}
\hline $\mathrm{PCl}$ score & Number of houses (\%) & $\begin{array}{l}\text { Houses positive for Aedes } \\
\text { larvae or pupae }(\%)\end{array}$ & Number of containers (\%) & Number of containers positive & $\begin{array}{l}\text { Number of } \\
\text { Aedes pupae }\end{array}$ & $\begin{array}{c}\text { Mean pupae per house } \\
\text { (SD, minimum-maximum) }\end{array}$ \\
\hline \multicolumn{7}{|c|}{ All time points combined ( $n=30$ clusters) } \\
\hline 3 & $30(1)$ & $12(40)$ & $179(1)$ & 32 & 991 & $33(145,0-791)$ \\
\hline 4 & $138(6)$ & $59(43)$ & $723(5)$ & 109 & 887 & $6(22,0-166)$ \\
\hline 5 & $623(26)$ & $250(40)$ & $3,548(26)$ & 431 & 5,739 & $9(105,0-2,580)$ \\
\hline 6 & $1,178(49)$ & $578(49)$ & $7,016(52)$ & 1,060 & 8,588 & $7(27,0-585)$ \\
\hline 7 & $327(14)$ & $167(51)$ & $1,610(12)$ & 283 & 1,450 & $4(12,0-97)$ \\
\hline 8 & $71(3)$ & 35 (49) & $283(2)$ & 56 & 286 & $4(13,0-81)$ \\
\hline 9 & $9(0)$ & $2(22)$ & $46(0)$ & 3 & 9 & $1(2,0-5)$ \\
\hline Missing & $24(1)$ & $11(46)$ & $124(1)$ & 18 & 49 & $2(5,0-16)$ \\
\hline Total & 2,400 (100) & 1,102 (46) & $13,529(100)$ & 1,992 & 17,999 & $7(60,0-2,580)$ \\
\hline \multicolumn{7}{|c|}{ October 2015 (heavy rain season): control at baseline ( $n=10$ clusters) } \\
\hline 3 & $3(1)$ & $1(33)$ & $18(1)$ & 2 & 11 & $4(6,0-11)$ \\
\hline 4 & $31(8)$ & $12(39)$ & $117(8)$ & 23 & 92 & $3(9.6,0-50)$ \\
\hline 5 & $126(32)$ & $51(40)$ & $483(31)$ & 78 & 594 & $5(15,0-129)$ \\
\hline 6 & $183(46)$ & $72(39)$ & 726 (47) & 105 & 759 & $4(11,0-82)$ \\
\hline 7 & 41 (10) & $18(44)$ & $142(9)$ & 28 & 205 & $5(12,0-59)$ \\
\hline 8 & $11(3)$ & 3 (27) & $33(2)$ & 7 & 3 & $0(1,0-3)$ \\
\hline 9 & $0(0)$ & $0(0)^{\prime}$ & $0(0)$ & 0 & 0 & - \\
\hline Missing & $5(1)$ & $4(80)$ & $25(2)$ & 4 & 12 & $2(5,0-12)$ \\
\hline Total & $400(100)$ & 161 (40) & $1,544(100)$ & 247 & 1,676 & $4(12,0-129)$ \\
\hline \multicolumn{7}{|c|}{ February 2016 (dry season) ( $n=10$ clusters) } \\
\hline 3 & $3(1)$ & $1(33)$ & $35(1)$ & 10 & 124 & $41(72,0-124)$ \\
\hline 4 & $14(4)$ & $6(43)$ & $169(5)$ & 10 & 98 & $7(24,0-91)$ \\
\hline 5 & $187(47)$ & $59(32)$ & $1,517(42)$ & 136 & 653 & $3(10,0-89)$ \\
\hline 6 & $161(40)$ & $62(39)$ & $1,584(44)$ & 167 & 947 & $6(19,0-131)$ \\
\hline 7 & $23(6)$ & $10(43)$ & $224(6)$ & 17 & 81 & $4(8,0-26)$ \\
\hline 8 & $6(2)$ & $3(50)$ & $46(1)$ & 8 & 18 & $3(7,0-18)$ \\
\hline 9 & $3(1)$ & $0(0)$ & $22(1)$ & 0 & 0 & $0(0,0-0)$ \\
\hline Missing & $3(1)$ & $1(33)$ & $23(1)$ & 2 & 0 & $0(0,0-0)$ \\
\hline Total & $400(100)$ & $142(36)$ & $3,620(100)$ & 350 & 1,921 & $5(16,0-131)$ \\
\hline \multicolumn{7}{|c|}{ June 2016 (light rain season) ( $n=10$ clusters) } \\
\hline 3 & $4(1)$ & $2(50)$ & $16(1)$ & 5 & 6 & $2(3,0-6)$ \\
\hline 4 & $32(8)$ & $20(63)$ & $152(6)$ & 33 & 272 & $9(22,0-121)$ \\
\hline 5 & $54(14)$ & $33(61)$ & 364 (15) & 53 & 607 & $11(29,0-148)$ \\
\hline 6 & 230 (58) & $174(76)$ & $1,480(61)$ & 342 & 2,741 & $12(35,0-330)$ \\
\hline 7 & 78 (20) & $52(67)$ & 411 (17) & 86 & 296 & $4(11,0-71)$ \\
\hline 8 & $2(1)$ & $1(50)$ & $5(0)$ & 1 & 0 & $0(0,0-0)$ \\
\hline 9 & $0(0)$ & $0(0)$ & $0(0)$ & 0 & 0 & - \\
\hline Missing & $0(0)$ & $0(0)$ & $0(0)$ & 0 & 0 & - \\
\hline Total & $400(100)$ & $282(71)$ & 2,428 (100) & 520 & 3,922 & $10(30,0-330)$ \\
\hline \multicolumn{7}{|c|}{ October 2016 (heavy rain season) ( $n=10$ clusters) } \\
\hline 3 & $4(1)$ & $1(25)$ & $40(2)$ & 2 & 32 & $8(16,0-32)$ \\
\hline 4 & $13(3)$ & $7(54)$ & 99 (4) & 15 & 115 & $9(26,0-92)$ \\
\hline 5 & $42(11)$ & $19(45)$ & $250(10)$ & 33 & 180 & $4(15,0-91)$ \\
\hline 6 & $280(70)$ & $96(34)$ & $1,698(70)$ & 146 & 807 & $3(9,0-96)$ \\
\hline 7 & $56(14)$ & $20(36)$ & 312 (13) & 30 & 98 & $2(5,0-25)$ \\
\hline 8 & $4(1)$ & $4(100)$ & $26(1)$ & 7 & 13 & $3(4,0-9)$ \\
\hline 9 & $1(0)$ & $0(0)$ & $4(0)$ & 0 & 0 & $0(0,0-0)$ \\
\hline Missing & $0(0)$ & $0(0)$ & $0(0)$ & 0 & 0 & - \\
\hline Total & 400 (100) & 147 (37) & 2,429 (100) & 233 & 1,245 & $3(10,0-96)$ \\
\hline
\end{tabular}

because the most productive containers were large water storage containers for animal husbandry that are not frequently changed or replaced. More wealthy families and those with nicer houses may be more likely to have lots of farm animals and, therefore, need these large water storage containers. In contrast, 30 premises $(2.5 \%)$ with the most adult female Aedes mosquitoes represented $25 \%$ of adult females and they tended to have relatively higher $\mathrm{PCl}$ scores (none had

TABLE 4

Association between each Premise Condition Index point and the mean density of Aedes adult females and pupae at household and cluster level over multiple seasons

\begin{tabular}{lll}
\hline & \multicolumn{1}{c}{ Adult Aedes } \\
\hline By household & & \\
$\quad$ Unadjusted & $1.25(1.11-1.39), P \leq 0.01$ & $0.74(0.57-0.96), P=0.02$ \\
$\quad$ Adjusted for survey & $1.16(1.02-1.31), P=0.02$ & \\
By cluster & & \\
Unadjusted & $1.80(1.12-2.88), P=0.01$ & $0.79(0.32-1.93), P=0.60$ \\
Adjusted for survey & $1.52(1.11-2.08), P=0.01$ & $0.78(0.35-1.73), P=0.55$ \\
\hline
\end{tabular}




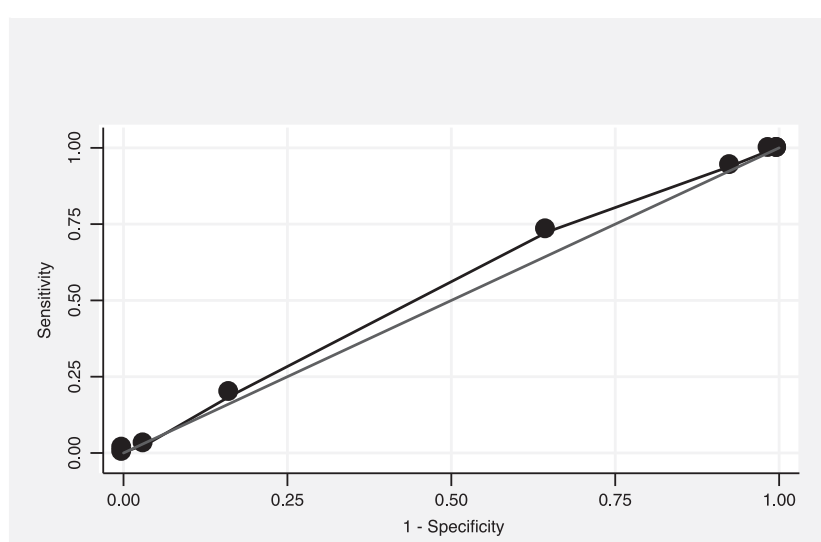

Area under $\mathrm{ROC}$ curve $=0.5389$

FIGURE 1. Receiver operating characteristic (ROC) curve of Premise Condition Index and prediction values in predicting the premises with the top quartile of adult mosquito density.

scores below 5). Therefore, the relative impact of one or two households has less weight on the overall measure with the adults than with immatures. Similar results have been found in other studies and resulted in affirmations of PCl's effectiveness and suggestions on how to incorporate it into national control programs. Similar positive associations in Mexico (odds ratio $[\mathrm{OR}]=1.27, P=0.001$ ) between $\mathrm{PCl}$ and Aedes larvae resulted in researchers concluding that the $\mathrm{PCl}$ can be an adequate estimator of the Ae. Agypti infestation rate. ${ }^{22}$ In Brazil, researchers found a positive correlation between $\mathrm{PCl}$ and houses positive for Aedes eggs $(r=0.97, P<0.01)$ and stated that the results clearly showed the usefulness of the method. ${ }^{23}$ They went one step further and suggested "in the case of dengue outbreaks, by having all representative house indices of the region, it will be much easier and less expensive to control the epidemic." Positive correlations between $\mathrm{PCl}$ and house positivity for larvae, pupae, and adult $A$. aegypti $(P>$ $0.05)$ led authors to advocate to the Brazilian Dengue Control Program the use of $\mathrm{PCl}$ to schedule the vector control teams' visits with different frequencies based on PCl scores. ${ }^{17}$ In Mexico, a significant positive correlation between average $\mathrm{PCl}$

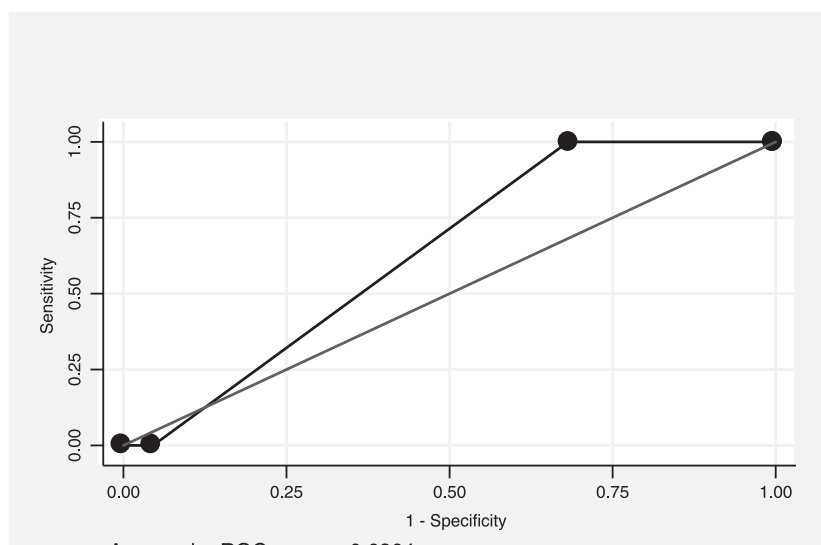

Area under ROC curve $=0.6364$

FIGURE 2. Receiver operating characteristic (ROC) curve of Premise Condition Index and prediction values in predicting the clusters with the top quartile of adult mosquito density.

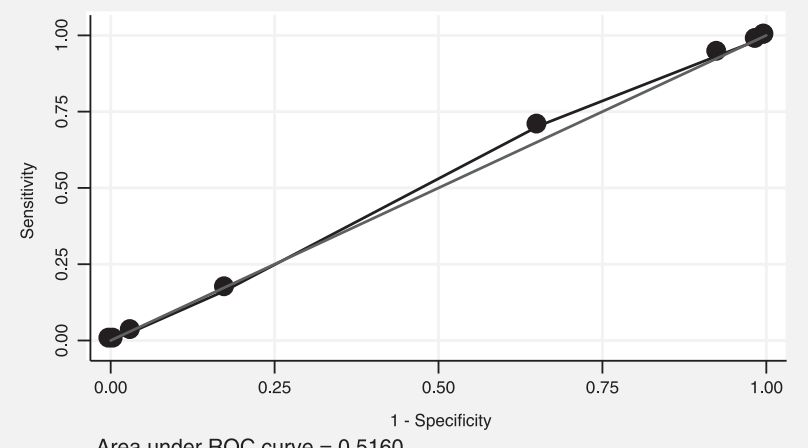

Area under ROC curve $=0.5160$

FIGURE 3. Receiver operating characteristic (ROC) curve of Premise Condition Index and prediction values in predicting the premises with the top quartile of immature mosquito density.

of a location and the house index was found (OR $=1.37, P=$ 0.007 ), and it was noted that in the near future, the authors expected to use information derived from $\mathrm{PCl}$ to "focalize integrated dengue vector control on houses/city blocks/ neighborhoods/areas with high levels of PCI (6-9)." 20 These examples show how relatively weak evidence has been used to advocate for PCl's use and integration into national policy.

However, finding statistically significant correlations does not always mean that the variables will be good predictors. ${ }^{35}$ In our study, ROC curves showed that PCI had "rather low accuracy" (AUC $=0.54$ and 0.52 , respectively) to predict premises in the top quartile for Aedes adult females and pupae. Additional ROC curves measuring the ability of $\mathrm{PCl}$ to predict clusters (as opposed to houses), which represent the top quartile of Aedes adult females and pupae also found it to have "rather low accuracy" (AUC $=0.64$ and 0.62, respectively). This is especially true when using highly variable outcomes such as immature measures. Therefore, control programs may want to use care when interpreting $\mathrm{PCl}$ associations in their area.

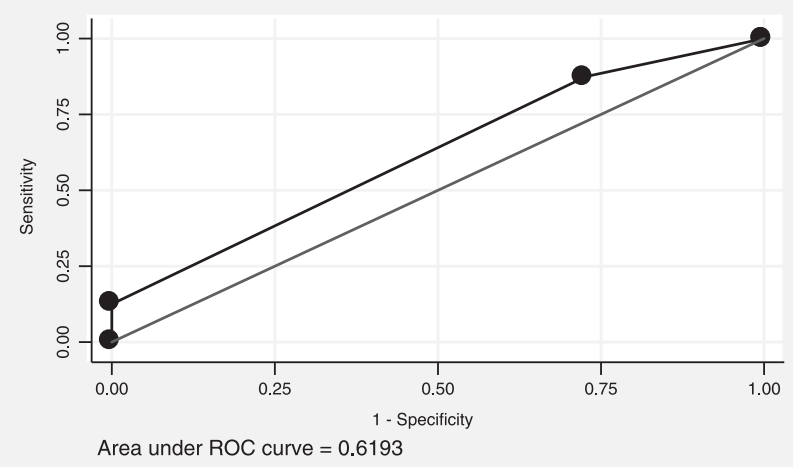

FIGURE 4. Receiver operating characteristic (ROC) curve of Premise Condition Index and prediction values in predicting the clusters with the top quartile of immature mosquito density. 
There are also several limitations of the $\mathrm{PCI}$ methodology to consider including that nonresidential premises, vacant lots, and construction worksites are often not ranked. Andrighetti et al. ${ }^{17}$ noted that $21 \%$ of the premises in their study could not be ranked and harbored $11.6 \%$ of larvae, $20.9 \%$ of pupae, and $20.8 \%$ of adults. In our study, we did not include vacant lots, schools, monasteries, or other public areas and, therefore, results may not be representative of those areas. In addition, the inability of the inspector to inspect or see into rear yards in some study settings may lead to misclassification. ${ }^{19}$ One of the key weaknesses that has been widely reported is that the scoring may not be standardized across individuals, teams, or organizations. ${ }^{19}$ One potential way to reduce this variability would be to use drones to take aerial photographs that could be scored by one individual or team. Another way could be to use $\mathrm{PCl}$ to classify geographical locations where it has been shown useful would be to assign one team to categorize the areas in known hot spots in advance of outbreaks. Then, the scores could be used to try to identify which hot spots or villages to target when resources are scarce. Nevertheless, it is unknown how use of $\mathrm{PCl}$ to prioritize households or geographical areas would be accepted within the communities. ${ }^{25}$ In addition, this would only work if $\mathrm{PCl}$ was not variable between seasons and years.

These results may not be generalizable to areas with more variability in housing conditions, different ecological conditions, or different mosquito abundance profiles. Considerable resources need to be invested in ensuring teams have standardized scoring of $\mathrm{PCl}$, the corresponding $\mathrm{PCl}$ cutoffs are followed correctly, and evaluating the acceptance of individuals or communities who are not prioritized. These resources may be better spent evaluating other methods to target premises or spent generally on Aedes control. Future studies could evaluate the use of $\mathrm{PCl}$ in other geographical settings, the effectiveness of $\mathrm{PCl}$ to identify premises with dengue infection, or the acceptance by the community of PCl's use where it is found to be effective.

Received June 14, 2019. Accepted for publication February 15, 2020.

Published online April 6, 2020.

Acknowledgments: We thank the government health staff, project volunteers, and participants of the study for all the support.

Financial support: The project was co-funded by the United Kingdom Agency for International Development (UKAID) (40097745) and the Deutsche Gesellschaft für Internationale Zusammenarbeit (GIZ) (81181153). UKAID funds funded project activities until March 2016 and GIZ funds funded activities until November 2016. N. A. and J. B. received support from the United Kingdom Medical Research Council (MRC) and Department for International Development (DFID) (MR/ K012126/1).

Disclaimer: The funders had no role in study design, data collection and analysis, decision to publish, or preparation of the manuscript. The views expressed in this article are those of the authors and do not necessarily reflect the official policy or position of the Department of Navy, Department of Defense, nor the U.S. Government.

Authors' addresses: John Hustedt, Malaria Consortium, Phnom Penh Center, Phnom Penh, Cambodia, and London School of Hygiene and Tropical Medicine. London, United Kingdom, E-mail: john.hustedt@ Ishtm.ac.uk. Dyna Doum, Vanney Keo, Sergio Lopes, and Jeffrey Hii, Malaria Consortium, Phnom Penh Center, Phnom Penh, Cambodia, E-mails: dynadoum@gmail.com, vanneykeo@gmail.com, sergiooolopes@ gmail.com, and j.hii@malariaconsortium.org. Ly Sokha, BunLeng Sam, and Leang Rithea, Cambodian National Dengue Control Program, Phnom Penh, Cambodia, E-mails: lysokha@yahoo.com, sambunleng@gmail.com, and rithealeang@gmail.com. Chan Vibol, World Health Organization, Phnom Penh, Cambodia, E-mail: chanv@who.int. Sebastien Boyer, Institut Pasteur du Cambodge, Phnom Penh, Cambodia, E-mail: sboyer@pasteur-kh.org. Marco Liverani, Neal Alexander, and John Bradley, London School of Hygiene and Tropical Medicine, London, United Kingdom, E-mails: marco.liverani@Ishtm.ac.uk, neal.alexander@Ishtm.ac.uk, and john.bradley@ Ishtm.ac.uk. Didot Budi Prasetyo and Agus Rachmat, US Naval Medical Research Unit-2, Phnom Penh, Cambodia, E-mails: didot.prasetyo.ctr@ namn2.org.kh and agus.rachmat.ctr@namru2.org.kh.

This is an open-access article distributed under the terms of the Creative Commons Attribution (CC-BY) License, which permits unrestricted use, distribution, and reproduction in any medium, provided the original author and source are credited.

\section{REFERENCES}

1. Seng CM, Setha T, Nealon J, Socheat D, Nathan MB, 2008. Six months of Aedes aegypti control with a novel controlled-release formulation of pyriproxyfen in domestic water storage containers in Cambodia. Southeast Asian J Trop Med Public Health 39: 822-826.

2. Vannice KS, Roehrig JT, Hombach J, 2015. Next generation dengue vaccines: a review of the preclinical development pipeline. Vaccine 33: 7091-7099.

3. Alphey L, Mckemey A, Nimmo D, Neira Oviedo M, Lacroix R, Matzen K, Beech C, 2013. Genetic control of Aedes mosquitoes. Pathog Glob Health 107: 170-179.

4. Franz AWE, Clem RJ, Passarelli AL, 2014. Novel genetic and molecular tools for the investigation and control of dengue virus transmission by mosquitoes. Curr Trop Med Rep 1: 21-31.

5. Ye YH, Carrasco AM, Frentiu FD, Chenoweth SF, Beebe NW, van den Hurk AF, Simmons CP, O'Neill SL, McGraw EA, 2015. Wolbachia reduces the transmission potential of dengueinfected Aedes aegypti. PLoS Negl Trop Dis 9: e0003894.

6. Turelli M, Barton NH, 2017. Deploying dengue-suppressing Wolbachia: robust models predict slow but effective spatial spread in Aedes aegypti. Theor Popul Biol 115: 45-60.

7. Bowman LR, Donegan S, McCall PJ, 2016. Is dengue vector control deficient in effectiveness or evidence?: systematic review and meta-analysis. PLoS Negl Trop Dis 10: e0004551.

8. Focks D, Alexander N, 2006. Multicountry Study of Aedes aegypti Pupal Productivity Survey Methodology: Findings and Recommendations.

9. Tun-Lin W, Kay BH, Barnes A, 1995. The Premise Condition Index: a tool for streamlining surveys of Aedes aegypti. Am J Trop Med Hyg 53: 591-594.

10. Seng CM, Setha T, Nealon J, Chantha N, Socheat D, Nathan MB, 2008. The effect of long-lasting insecticidal water container covers on field populations of Aedes aegypti (L.) mosquitoes in Cambodia. J Vector Ecol 33: 333-341.

11. Chadee DD, 2004. Key premises, a guide to Aedes aegypti (Diptera: Culicidae) surveillance and control. Bull Entomol Res 94: 201-207.

12. Stewart Ibarra AM, Ryan SJ, Beltran E, Mejia R, Silva M, Munoz A, 2013. Dengue vector dynamics (Aedes aegypti) influenced by climate and social factors in Ecuador: implications for targeted control. PLoS One 8: e78263.

13. Kay BH, Ryan PA, Lyons SA, Foley PN, Pandeya N, Purdie D, 2002. Winter intervention against Aedes aegypti (Diptera: Culicidae) larvae in subterranean habitats slows surface recolonization in summer. J Med Entomol 39: 356-361.

14. Williams CR, Long SA, Webb CE, Bitzhenner M, Geier M, Russell RC, Ritchie SA, 2007. Aedes aegypti population sampling using BG-Sentinel traps in north Queensland Australia: statistical considerations for trap deployment and sampling strategy. $\checkmark$ Med Entomol 44: 345-350.

15. Knox TB, Yen NT, Nam VS, Gatton ML, Kay BH, Ryan PA, 2007. Critical evaluation of quantitative sampling methods for Aedes aegypti (Diptera: Culicidae) immatures in water storage containers in Vietnam. J Med Entomol 44: 192-204.

16. Maciel-de-Freitas R, Peres RC, Souza-Santos R, Lourenco-deOliveira R, 2008. Occurrence, productivity and spatial distribution of key-premises in two dengue-endemic areas of Rio de 
Janeiro and their role in adult Aedes aegypti spatial infestation pattern. Trop Med Int Health 13: 1488-1494.

17. Andrighetti MTM, Galvani KC, Macoris MDLDG, 2009. Evaluation of premise condition index in the context of Aedes aegypti control in Marília, São Paulo, Brazil. Dengue Bull 33: 167-175.

18. Azil AH, Bruce D, Williams CR, 2014. Determining the spatial autocorrelation of dengue vector populations: influences of mosquito sampling method, covariables, and vector control. $J$ Vector Ecol 39: 153-163.

19. Basker P, Ezhil R, 2012. Study on the correlation of premises condition index and the presence of larvae of Aedes species mosquitoes in human dwellings of the cuddalore district of Tamil Nadu, India. Osong Public Health Res Perspect 3: 3-7.

20. Manrique-Saide $P$ et al., 2013. The risk of Aedes aegypti breeding and premises condition in South Mexico. J Am Mosq Control Assoc 29: 337-345.

21. Moloney JM, Skelly C, Weinstein P, Maguire M, Ritchie S, 1998. Domestic Aedes aegypti breeding site surveillance: limitations of remote sensing as a predictive surveillance tool. Am J Trop Med Hyg 59: 261-264.

22. Espinoza-Gómez F, Moisés Hernández Suárez C, Coll Cárdenas $\mathrm{R}, 2001$. Factors that modify the larval indices of Aedes aegypti in Colima, Mexico. Rev Panam Salud Publica 10: 6-12.

23. Nogueira LA, Gushi LT, Miranda JE, Madeira NG, Ribolla PE, 2005. Application of an alternative Aedes species (Diptera: Culicidae) surveillance method in Botucatu city, Sao Paulo, Brazil. Am J Trop Med Hyg 73: 309-311.

24. Kay HB, Watson TM, Ryan PA, 2008. Definition of productive Aedes notoscriptus (Diptera: Culicidae) habitats in western Brisbane, and a strategy for their control. Aust J Entomol 47: 142-148.

25. Peres RC, Rego R, Maciel-de-Freitas R, 2013. The use of the premise condition index $(\mathrm{PCl})$ to provide guidelines for Aedes aegypti surveys. J Vector Ecol 38: 190-192.
26. Montgomery B, Ritchie S, 2002. Roof gutters: a key container for Aedes aegypti and Ochlerotatus notoscriptus (Diptera: Culicidae) in Australia. Am J Trop Med Hyg 67: 244-246.

27. Trewin BJ, Kay BH, Darbro JM, Hurst TP, 2013. Increased container-breeding mosquito risk owing to drought-induced changes in water harvesting and storage in Brisbane, Australia. Int Health 5: 251-258.

28. Huy $R$ et al., 2010. National dengue surveillance in Cambodia 1980-2008: epidemiological and virological trends and the impact of vector control. Bull World Health Organ 88: 650-657.

29. WHO, 2015. Dengue Situation Update 462 Update on the Dengue Situation in the Western Pacific Region Northern Hemisphere. Geneva, Switzerland: World Health Organization.

30. World Health Organization, 2009. Dengue Guidelines for Diagnosis, Treatment, Prevention and Control, New Edition. Geneva, Switzerland: World Health Organization. Available at: http://whqlibdoc.who.int/publications/2009/9789241547871_ eng.pdf.

31. Hustedt $J$ et al., 2017. Determining the efficacy of guppies and pyriproxyfen (Sumilarv ${ }^{\circledR} 2 \mathrm{MR}$ ) combined with community engagement on dengue vectors in Cambodia: study protocol for a randomized controlled trial. Trials 18: 367

32. Espinoza-Gómez F, Hernández-Suárez CM, Coll-Cárdenas R, 2001. Educational campaign versus malathion spraying for the control of Aedes aegypti in Colima, Mexico. Public Health Pol Pract 56: 148-152.

33. Zeileis A, Lumley T, Berger S, Graham N, 2018. Robust Covariance Matrix Estimators. Available at: https://cran.rproject.org/web/packages/sandwich/sandwich.pdf. Accessed February 7, 2018

34. Swets JA, 1988. Measuring the accuracy of diagnostic systems. Science 240: 1285-1293.

35. Shmueli G, 2010. To explain or to predict? Stat Sci 25: 289-310. 